\title{
Research on Multi-Storey Indoor Wifi Positioning Algorithm based on Image Recognition
}

\author{
Hao DING ${ }^{1, a}$,Lei WANG ${ }^{2, b}$, Ya-Nan LIU ${ }^{3, C}$,Jing-Qi DENG ${ }^{4, d}$, Ming-Jun ZHAO ${ }^{5, e}$ \\ ${ }^{1}$ Jilin University, Jilin, Changchun,China \\ 2Jilin University, Jilin, Changchun,China \\ ${ }^{3}$ Jilin University, Jilin, Changchun,China \\ ${ }^{4}$ Jilin University, Jilin, Changchun,China \\ 5 Jilin University, Jilin, Changchun,China

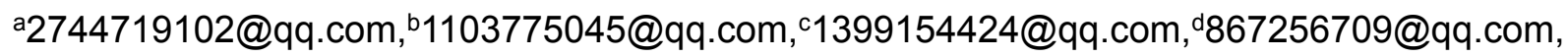 \\ e805153853@qq.com
}

\begin{abstract}
Keywords: fingerprint; storey discrimination; indoor positioning; map information; image recognition

Abstract.To address the inaccuracy of multi-storey indoor positioning, the paper proposes a method involving $\mathrm{WiFi}$ fingerprint positioning based on the prior information of map environment and position correction based on the image recognition technique. First, we establish a model of map environment information at the off-line stage, then identify the storey using the storey discrimination algorithm of the RSSI threshold value, and finally compute the approximate position of the terminal in combination with the map information model. Meanwhile, the image recognition technique is employed to correct the terminal position. Compared with the traditional fingerprint positioning method, the indoor positioning technique can not only improve the positioning accuracy but also reduce the complexity of the fingerprint matching computation at the on-line stage.
\end{abstract}

\section{Introduction}

With the rapid development of the mobile internet and the popularization of the mobile terminals, the mobile users have an increasing demand for real-time and accurate position information. The Location Based Service (LBS) attracts wide attention from the public as a result of its broad prospects in the civil, commercial, military, and emergency rescue applications. Currently, the most majority of the domestic and international LBS applications use the GPS satellite positioning. The GPS receiver may have decreased positioning accuracy and even cannot complete the positioning process when operating in a city with crowed buildings or indoor due to the fact that the signal intensity attenuates considerably under the influence of buildings, walls etc. At present, the smart phones are widely used and WiFi also basically covers most public places. The WiFi positioning has an advantage over various other positioning methods as it requires no additional requirements for hardware and has such characteristics as low power consumption, high accuracy etc. As a highlight in research on indoor positioning techniques in recent years, the WiFi-based indoor positioning technique can identify the users' positions using the signal received by the mobile terminal from the wireless access point (AP), improve the utilization rate of the existing equipment, and achieve convenient and efficient positioning service at a low cost. In terms of the WiFi indoor positioning technique, the fingerprint positioning technique based on the Received Signal Strength Indicator (RSSI) is widely used due to its low requirements for equipment and simple operation compared with the methods based on the time of arrival (TOA), Angle of Arrival (AOA) etc. The measured value of on-line receiving RSSI at the terminal substantially deviates from the off-line fingerprint database under the influence of the time-variant characteristics of the WiFi signal propagation and the environmental factors. In addition, general WiFi fingerprint positioning computation requires traversal of the fingerprints of all reference points. It leads to an increased number of fingerprints in the positioning system and significantly increased complexity of matching computation when applied in a multi-storey environment. Research indicates that the prior information of the 
positioning regional environment can improve the positioning accuracy by reducing the signal non-line-of-sight (NLOS) deviation during signal propagation due to the obstacles.[1-3]To deal with the above problems, the paper proposes a WiFi fingerprint positioning method based on the prior information of the map environment. First, we establish a statistical model of map environment information and perform off-line training of the fingerprints with the affinity propagation clustering algorithm for reducing the computation complexity at the positioning stage. Furthermore, to cope with the low accuracy rate of storey discrimination caused by height error in WiFi positioning, the paper proposes a storey discrimination algorithm based on the RSSI threshold value for improving the accuracy rate of multi-storey positioning. The position estimation algorithm based on the map information model is utilized to reduce the effects of NLOS deviation, interference, noise etc. Finally, the image recognition technique is used to correct the terminal position and improve the WiFi positioning accuracy and stability.

\section{WiFi Indoor Positioning System Based on the Map Environment Information}

\section{Fingerprint positioning method}

There are many positioning techniques based on WiFi wireless network. The positioning technique based on the signal intensity RSSI is most common. The location fingerprint positioning method is common in the RSSI positioning technique. The WiFi fingerprint positioning includes two stages, off-line stage and on-line stage. At the data collection stage, $\mathrm{N}$ data collection points are uniformly selected within the region. The RSSI information of all AP at each collection point is collected. All RSSI information and the coordinates of the point $(x, y)$ are stored in the database for calling at the second stage. At the real-time positioning stage, a user may make a request for positioning in an unknown position. The Android mobile phone will collect the RSSI information of AP at this point and transfer the information to the database. The approximate position coordinates $(\mathrm{x}, \mathrm{y})$ are obtained through the matching algorithm and the RSSI data collected at the first stage and fed back to the user for completion of positioning. The operation steps at each stage are detailed below.

$\mathrm{N}$ reference points in the positioning region are first identified, i.e. $i \in\{1,2, \ldots, \mathrm{N}\}$. Then, these reference points are traversed for RSSI sampling. The fingerprint of each reference point is recorded as $\mathrm{FPi}=(\mathrm{MACm}, \mathrm{RSSIi}), \mathrm{m} \in\{1,2, \ldots, \mathrm{n}\}$. MACm represents the MAC address of the mth AP searched by the current reference point. Finally, the RSSI of each reference point is sampled and average repeatedly and assigned in the same group with its position coordinates $(\mathrm{x}, \mathrm{y}, \mathrm{z})$ to form a fingerprint map (radio map, RM). To facilitate computation and reduce the effect of AP with weak signal on the computational result, the RSSI is set to the minimum value $-110 \mathrm{dBm}$.

During positioning at the second stage, the existing fingerprint matching algorithm can be divided into certainty matching algorithm (such as K- nearest neighbor algorithm, KNN) and the probability matching algorithm (such as the maximum posteriori estimate MAP or the minimum root-mean-square error, MMSE). The KNN algorithm is widely used as a typical certainty matching algorithm. The Euclidean distance between the fingerprint of the existing signal and the fingerprints of various reference points in the fingerprint database is computed to select $\mathrm{k}$ reference points with the shortest distance to the existing fingerprint. The average value of their coordinates served as the terminal position [4].

\section{kNN algorithm (K-Nearest Neighbor algorithm) [5]}

The core idea of the near algorithm, i.e K-nearest sorting algorithm (kNN, k-NearestNeighbor) is as follows: If a sample belongs to a category in most of the $\mathrm{k}$ nearest samples in the characteristic space, then the sample would also belong to the category and have the characteristics of the sample in the category. The value of parameter $\mathrm{k}$ in the KNN algorithm should be selected accurately. The category of the reference point cannot be determined accurately, and the error is increased when $\mathrm{k}$ is small, that is to say, the number of the near points is small. Farther points would be selected thus leading to inaccurate positioning results when $\mathrm{k}$ is large. Thus, the value of parameter $\mathrm{k}$ should be determined by repeated measurements. APn represents $n \mathrm{WiFi}$ hotspots in the positioning region. At 
the data collection stage, the RSSI of $\mathrm{n}$ WiFi hotspots is collected at the ith position, $\mathrm{i}=1,2$, $3, \ldots, \mathrm{m} . \mathrm{m}$ is the number of the reference points. The RSSI values collected at each WiFi hotspot are averaged. Rij is the average value of RSSI at the $\mathrm{jth}$ WiFi in the ith position, $\mathrm{j}=1,2,3, \ldots, n ; n$ is the number of WiFi hotspots, $(\mathrm{xi}, \mathrm{yi})$ is the actual coordinate of Point $\mathrm{i}$. At the positioning stage, the average value of RSSI of the jth WiFi hotspot at the point to be positioned is $\mathrm{R}_{\mathrm{j}}$. The distance between $R_{i j}$ and $R_{j}$ is computed as follows.

$$
\mathrm{d}_{j}=\sqrt{\sum_{i=1}^{n}\left(R_{j}-R_{i \mathrm{j}}\right)^{2}}
$$

In the $\mathrm{kNN}$ algorithm, $\mathrm{k}$ points with the smallest $\mathrm{d}_{\mathrm{j}}$ are selected as the reference points for positioning the point to be located. The coordinate of the point to be located is the center of mass of the $\mathrm{k}$ reference points [7]:

$$
(x, y)=\frac{1}{k} \sum_{i=1}^{k}\left(x_{i}, y_{i}\right)
$$

where, $\left(\mathrm{x}_{\mathrm{i}}, \mathrm{y}_{\mathrm{i}}\right)$ is the coordinate of the ith reference point.

\section{Modeling of Indoor Map Environment}

For WiFi positioning with the prior information of the map environment, it is required to establish a mathematical model of the map environment, including: positioning regional model, AP covering region model etc.

In the positioning regional model, $\mathrm{R}$ is the positioning region in the map; the positioning region $\mathrm{R}$ is the blank part, and the black part represents the map boundary and wall, as shown in the map. The region to be positioned $\mathrm{R}$ can be obtained by map modeling. Such that, no users will be positioned to the non-positioning region, i.e. the black region in the map.

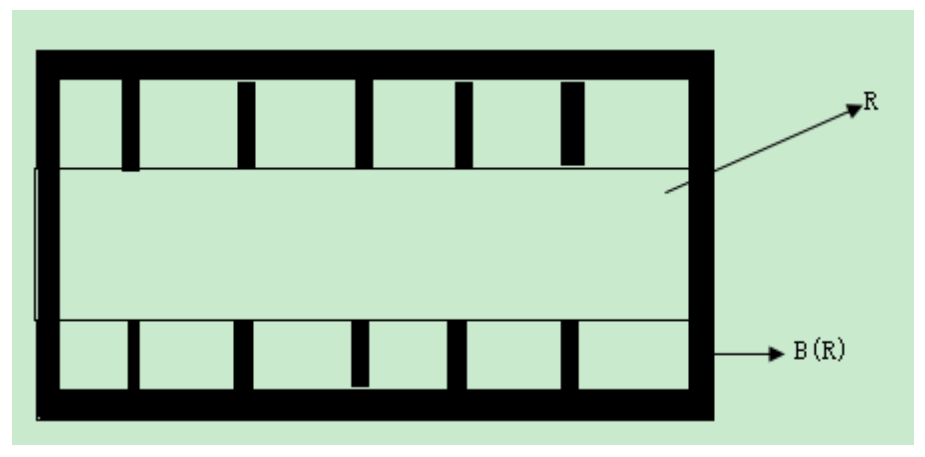

If the two-dimensional coordinate of the terminal position is $\mathrm{p}=[\mathrm{x}, \mathrm{y}]^{\mathrm{T}}$, based on the uniform probability density function, it can be expressed as:

$$
\mathrm{f}(p)= \begin{cases}1 / A R & , \mathrm{p} \in \mathrm{R} \\ 0 & , \text { non - positioning region }\end{cases}
$$

where, $\mathrm{R} \in \mathrm{p}, \mathrm{R}$ is the positioning area in the map; $\mathrm{AR}_{\mathrm{R}}$ is the area of the region. The formula describes the map model of uniform distribution, including only one variable $\mathrm{R}_{\mathrm{A}}$, that is to say, the probability density function is completely described by $\mathrm{R}$. As shown in the above map, the positioning region $R$ is the blank part, and $B(R)$ is the map boundary. The region to be located $R$ can be obtained by map modeling. Such that, no users will be positioned to the non-positioning region, i.e. the shadow area in the map.

\section{On-line Positioning Stage}

\section{Storey discrimination $[8]$}

Research indicates that the signal intensity may experience significant attenuation when the WiFi signal passes through the wall due to the fact that the metallic reinforced concrete is usually used in the wall between storeys $(15-20 \mathrm{dBm})$. [9] The phenomenon indicates that: 1) The RSSI of AP of 
the same storey is stronger than that of other storeys when the physical distance is equal. 2) In accordance with the triangular theorem, the distance between the terminal and the AP of the same storey is shorter the planar projections of different APs, i.e. coordinates ( $x, y)$ are the same. In conclusion, the RSSI of AP of other storeys is significantly smaller than that of the same storey when the plane positions, i.e. coordinates $(\mathrm{x}, \mathrm{y})$ are the same. The differences in RSSI between storeys are used to set the threshold value $\mathrm{R}_{0}$. rFN, the APs within the threshold value of various storeys is counted. The Storey $\mathrm{F}_{\mathrm{r}}$ corresponding to the maximum value of $\mathrm{r}_{\mathrm{FN}}$ is returned. $\mathrm{F}_{\mathrm{r}}$ is the storey of the current terminal.

\section{Positioning stage}

Ideally, we can determine the position of the point to be located based on the distance from the point to be located to the three APs obtained at the distance measuring stage. Circles are described with 3 APs of known position as the centers and the distances from the centers to the points to be located as the radii. The intersection point of these circles is the position of the point to be located [10].

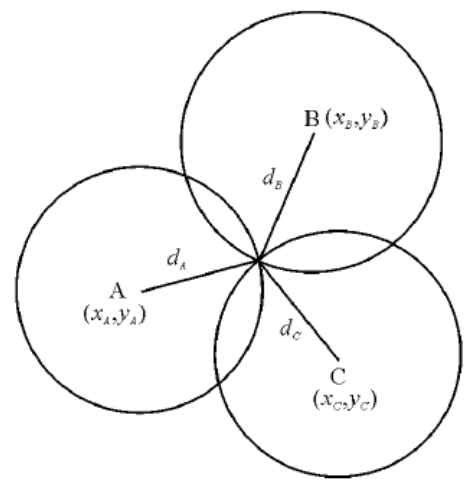

Figure 1: Point A, Point $\mathrm{B}$ and Point $\mathrm{C}$ are three WiFi hotspots respectively. The straight-line distances from Point $\mathrm{A}$, Point $\mathrm{B}$, and Point $\mathrm{C}$ to the mobile phone are $\mathrm{dA}, \mathrm{dB}$, and $\mathrm{dC}$, with three points as the center of the circle,distance di as the radius, and three circles meet at Point $\mathrm{D}$.

As shown in Figure 1, it is assumed that the coordinate of the unknown node, D, i.e. the intersection point of the three circles, is $(\mathrm{x}, \mathrm{y})$. The coordinates of Point $\mathrm{A}$, Point $\mathrm{B}$, and Point $\mathrm{C}$ are $\left(\mathrm{xA}, \mathrm{yA}_{\mathrm{A}}\right),(\mathrm{xB}, \mathrm{yB})$, and $(\mathrm{xC}, \mathrm{yC})$. The distances from Point $\mathrm{A}$, Point $\mathrm{B}$, and Point $\mathrm{C}$ to Point $\mathrm{D}$ are $\mathrm{d}_{\mathrm{A}}$, $\mathrm{d}_{\mathrm{B}}$, and $\mathrm{dc}$. Based on the distance equation, we can obtain the following equation sets:

$$
\left\{\begin{array}{l}
\mathrm{d}_{\mathrm{A}}=\sqrt{\left(x_{A}-x\right)^{2}+\left(y_{A}-y\right)^{2}} \\
\mathrm{~d}_{B}=\sqrt{\left(x_{B}-x\right)^{2}+\left(y_{B}-y\right)^{2}} \\
\mathrm{~d}_{C}=\sqrt{\left(x_{C}-x\right)^{2}+\left(y_{C}-y\right)^{2}}
\end{array}\right.
$$

\section{Position Correction Stage}

The WIFI indoor positioning and the KNN algorithm of the position fingerprint will roughly limit the user's position to a smaller region. It is assumed that two near points, Point A and Point B (at which the user is actually located), are partitioned by a wall. The positioning algorithm positions it to Point A due to some uncontrollable interference factors. A wrong path may be provided to the users if no correction measures are taken. We expect that the system can accurately judge the existing positions of users and provide reliable paths to the destination when they open the camera of their mobile devices.

We propose a solution for image recognition. In terms of the off-line data collection, 360-degree image information is collected at each sampling point. It is transformed into binary image and 
stored in the database by generating reasonable threshold values (enabling the static things such as doors, windows etc. on the image to have obvious and clear edges). In the case of occurrence of near regions, e.g. Point $\mathrm{A}$ and Point $\mathrm{B}$ as mentioned above, the image identification degree is too low to distinguish between $\mathrm{A}$ and $\mathrm{B}$ thus leading to positioning errors. Thus, we should find a method to distinguish between the near and similar regions. A marker is added to the near and similar regions for distinguishing. The marker is a LED lamp. The LED lamp has the two major advantages: (1) low price; (2) ease of identification of the processed binary images with obvious edges.

\section{Binary processing}

The collected images of the LED lamp are subject to binary processing. The optimal iterative threshold binarization segmentation is used. The principle of the optimal iterative threshold method is detailed as follows. Two probability density functions of normal distribution are used to approximate the histogram obtained by the image, and compute the error probability of segmentation. The error probability includes two parts. One is the error probability to classify the background pixel points as foreground pixel points. The other is the error probability to classify the foreground pixel points to the back ground pixel points. If there is gray value $\mathrm{T}$ that makes the error probability smallest, it would be the threshold. The $\mathrm{T}$ value can achieve image segmentation with the minimum error [11].

The $T$ value can be obtained with the iterative algorithm.

Two initial values $\mathrm{a}_{1}$ and $\mathrm{a}_{2}$ are given arbitrarily. Both are integers between 0 and 255 (with the gray value assumed to be between 0 and 255). We can obtain the initial threshold value:

$$
\mathrm{T}_{0}=\left(\mathrm{a}_{1}+\mathrm{a}_{2}\right) / 2
$$

(2) The image is partitioned into two parts by the threshold value. Then, the average gray values $b_{1}$ and $b_{2}$ of both parts are computed respectively. The average value $T_{x}=\left(b_{1}+b_{2}\right) / 2$ of $\quad b_{1}$ and $\mathrm{b}_{2}$ is computed. $\mathrm{T}_{\mathrm{x}}$ will be the threshold value if $\mathrm{T}_{0}$ and $\mathrm{T}_{\mathrm{x}}$ approximate sufficiently. Otherwise, Step (2) will start if $T_{0}=T_{x}$.

It is thus clear that the iterative algorithm is always convergent whatever the initial value is. The gray value with a gray value distribution probability of 0 should not affect the binaryzation process when the optimal iteration method is used. Thus, the range of the initial values of $a_{1}$ and $a_{2}$ can be further reduced. $a_{1}$ is the minimum gray value $i$ with the gray value distribution probability $P_{i}$ not equal to $0 . a_{2}$ is the maximum gray value $j$ with the gray value distribution probability $P_{j}$ not equal to 0 . In addition, the control accuracy of convergence is selected according to the below principle. $\mathrm{T}_{0}$ and $\mathrm{T}_{\mathrm{x}}$ are considered to approximate sufficiently if the absolute value of their difference is smaller than 1. Two cases may occur when the difference of two floating-point numbers is smaller than 1. One is that the round-off numbers of the two floating-point numbers are equal; the other is that one integer is between the two floating-point numbers. Thus, we select the larger floating-point number for rounding thus reducing the unexpected errors due to rough control accuracy. This is the threshold value of the optimal iteration method [11].

If the original image is:

$$
f(x, y)=\left[\begin{array}{llllll}
x_{11} & x_{12} & \ldots & x_{1 j} \\
x_{21} & x_{22} & \ldots & x_{2 j} \\
\ldots & \ldots & & \ldots & \\
x_{i 1} & x_{i 2} & \ldots & x_{i j}
\end{array}\right],
$$

the partitioned image is:

$$
g(x, y)=\left\{\begin{array}{cc}
0 & \mathrm{f}(\mathrm{x}, \mathrm{y})<\mathrm{T}_{\mathrm{x}} \\
1 & \mathrm{f}(\mathrm{x}, \mathrm{y}) \geq \mathrm{T}_{\mathrm{x}}
\end{array}\right.
$$

\section{Rectangular partitioning code for edge matching}

Most information of an image exists in the the image edge and present as discontinuity of the local 
image characteristics, i.e. the place with drastic changes in gray value. Thus, the edge is defined as the regional boundary with drastic changes in gray value [12].

[13] proposes the rectangular partitioning coding rules for edge matching and improve the image compression effect. The major concept of the rectangular partitioning coding rules for edge matching is to first detect the image edge points. Then, the images are subject to non-overlapping rectangular partitioning. Based on the partitioned rectangles and the adjacent edge points, the pixel values of the edge points are changed to maximize the area of the rectangle.

Based on theoretical computation, the compression effect of the rectangular partitioning codes for edge matching improves by approximately $20 \%$ compared with that of the traditional rectangular partitioning codes. No significant differences are found between the two codes in images with regular edges.

Such that, the actual characteristics of various collection points are collected by means of off-line data collection.

\section{Summary}

The images obtained by the camera are subject to binarization processing and compared with the image of the region near the WIFI positioning point after the user starts the camera of a mobile device. The device will provide a reasonable route map after it verifies the accurate position and face orientation of the user.

\section{Reference}

[1] DAVIDSON P, COLLIN J, TAKALA J. Application of particle filters for indoor positioning using floor plans[C]// Ubiquitous Positioning Indoor Navigation and Location Based Service. Kirkkonummi, Finland: IEEE, 2010.

[2] ZHOU rui . Application of indoor structure layout to improve Wi-Fi positioning accuracy and stability[J]. Journal of University of Electronic Science and Technology of China , 2013, 42(2): 295-299.

[3] SANGWOOK B, SEOKSEONG J, YU Chan-su, et al. Characteristics of a large-scale WiFi radiomap and their implications in indoor localization[C]//International Conference on Network of the Future. Paris, France: IEEE, 2013.

[4] HUANG Yung-fa,ZHENG You-ting. Performance of an MMSE based indoor localization with wireless sensor networks[C]//International Conference on Networked Computing and Advanced Information Management (NCM). Gyeongju, South Korea: IEEE, 2010.

[5] HARA S, ANZAI D. Use of a simplified maximum likelihood function in a wlan-based location estimation[C]// IEEE Conference on Wireless Communications and Networking. Budapest, Hungary: IEEE, 2009

[6] YANG Z,WU C,LIU Y.Locating in fingerprint space: wireless indoor localization with little human intervention[C]. Proceedings of the 18th Annual International Conference on Mobile Computing and Networking.ACM,2012 : 269-280.

[7] ZHANG xiao-liang, ZHAO ping, XU guan-qing, etc. Research and application of indoor location based on an optimized KNN algorithm[J].International Electronic Elements | Int Electr Elem , 2013,21(7) : 44-46.

[8] LI hai-tao, QI shuang . Research on multi floor WiFi positioning technology based on indoor map environment information[J]. Journal of University of Electronic Science and Technology of China ,2017,1(46):35-36. 
[9] WU C S, ZHENG Y, LIU Y H, et al. WILL: Wireless indoor localization without site survey[J]. IEEE Transactions on Parallel and Distributed Systems, 2013, 24(4): 64-72

[10] National Instruments. The PXI system architcture [DB/OL].National Instruments,1997.

[11] ZHANG qi,Image Binarization Algorithm Combined with Edge Detection[D].Jilin University, 2011:10-11

[12] DUAN rui-ling, LI qing-xiang, LI yv-he . Review of image edge detection methods[J]. Optical technique, 2005,(03):415-419.

[13] JIANG hai-bo. Improved rectangle division coding of Binary Image based on edge detection[A]. Chinese society of Electronics.2010:5. 